\title{
Los procesos de estrés laboral y desgaste profesional (burnout): diferenciación, actualización y líneas de intervención
}

\section{Stress and burnout at work: differences, updates and lines of intervention}

\author{
Raquel Rodríguez Carvajal, Sara de Rivas Hermosilla \\ Facultad de Psicología, Universidad Autónoma de Madrid. España.

\section{Correspondencia:} \\ Raquel Rodríguez Carvajal \\ Universidad Autónoma de Madrid \\ Facultad de Psicología. Despacho 35. Módulo I \\ c/ Ivan Paulov, 6 \\ 28049 Madrid. España. \\ Tfno.: +34914975122 \\ E-mail: raquel.rodriguez@uam.es
}

ResUmen

El escenario laboral actual marcado por la globalidad y la inestabilidad genera unas condiciones que hace que muchos profesionales experimenten frustración y un alto nivel de tensión emocional, que conlleva un aumento de los niveles de estrés laboral y del desgaste profesional. La importancia del estudio del estrés laboral y el burnout ha incrementado en los últimos años la productividad científica en estos temas. Se ha realizado por ello una revisión de la literatura para ofrecer una visión global y actualizada de ambos procesos. Se muestran los principales modelos y las variables de estudio vigentes así como las principales líneas más actuales de intervención que se vienen desarrollando hasta la fecha.

Palabras clave: Estrés Psicológico; Agotamiento Profesional; Estudios de Intervención.

\begin{abstract}

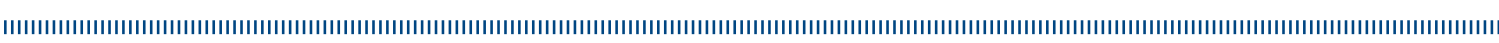

Today's workplace is constantly changing. Organizations are confronted with multiple challenges, brought by globalization and instability. This situation causes many professionals to experience frustration and a high level of emotional tension, which may easily lead to increased levels of occupational stress and burnout. The importance of studying occupational stress and burnout has led to an increase in the scientific productivity in these areas during the last years. The present literature review provides an overview and update of both occupational stress and burnout processes. The main models are presented as well as the current variables in study. The main lines of intervention that have been developed to date are also reviewed.
\end{abstract}

Keywords: Psychological Stress; Burnout; Intervention Studies. 


\section{INTRODUCCIÓN}

En el escenario laboral que nos encontramos, marcado por la globalidad y la inestabilidad, se observa un alto nivel de complejidad. Es un escenario dinámico, de fusiones empresariales, reingeniería de recursos humanos y desarrollo tecnológico exponencial, donde prima la necesidad de reducción de costes, la demanda en el aumento de la productividad y de la satisfacción del cliente ${ }^{1}$. Estas presiones laborales se ven además afectadas por otros aspectos sociales que también han cambiado y que suponen un añadido más a este entramado, como por ejemplo un menor nivel de autonomía, una mayor sobrecarga debido a la reducción de personal, el aumento de las demandas emocionales, o que los objetivos del trabajo no estén bien definidos, no sean muy claros o no se reciba feedback sobre ellos ${ }^{2}$.

Todos estos cambios están llevando a una serie de consecuencias tanto personales, principalmente en materia de salud de la población trabajadora, como económicas. Según los datos de la Agencia Europea de Seguridad y Salud en el trabajo, en el año 2009 se estimaba una pérdida de 1.250 millones de días de trabajo anuales debidos a problemas de salud relacionados con el trabajo. Por su parte, el Consejo de Seguridad de Estados Unidos, cifra dicha pérdida en 183 billones de dólares anuales en 2010, lo que conlleva que cada trabajador debe incrementar su productividad una media de 1.300 dólares para que se pueda suplir dicho déficit. Esta pérdida de recursos debido al creciente aumento de los problemas de salud, pérdida de días de trabajo, bajas laborales, absentismo y abandono, conllevan un coste directo a todos los niveles.

Y es que en estas condiciones en las que el trabajo no facilita la integración y el desarrollo del individuo, o cuando se lleva a cabo en condiciones precarias, hace que muchos profesionales experimenten frustración y un alto nivel de tensión emocional, que conlleva un aumento de los niveles de estrés laboral y de desgaste profesional. En una reciente investigación llevada a cabo en más de 2.000 personas, trabajadores de diversos sectores tanto públicos como privados ${ }^{3}$, se observó que el $41 \%$ informaba de altos niveles de estrés laboral. Dos de cada tres indicaban sentir un aumento significativo de sobrecarga de trabajo, y un 36\% un menor apoyo por parte de compañeros y supervisores. Además, uno de cada cinco no informaba sobre su alto nivel de estrés y un $48 \%$ evitaba cogerse días de baja por enfermedad, ambos por miedo al despido.

Dada la situación actual, la necesidad del estudio de los procesos de estrés y de desgaste profesional se hace más patente, y la producción científica da muestra de ello. Atendiendo a la evolución del número de investigaciones llevadas a cabo desde el año 2002 según la base de datos PsycInfo, se puede observar una alta productividad ya en los inicios del siglo xxi del estrés laboral, junto con un aumento sistemático a lo largo de los últimos años en el estudio del burnout o desgaste profesional (ver figura 1). Es importante señalar que el descenso en el año 2011 es meramente artefactual en la medida en que aún no están recogidas en las bases de datos las publicaciones de los últimos 4 o 5 meses del presente año.

Figura 1. Evolución del número de publicaciones cientificas sobre estrés laboral y burnout desde el 2002 al 2011 según la base de datos Psyclnfo

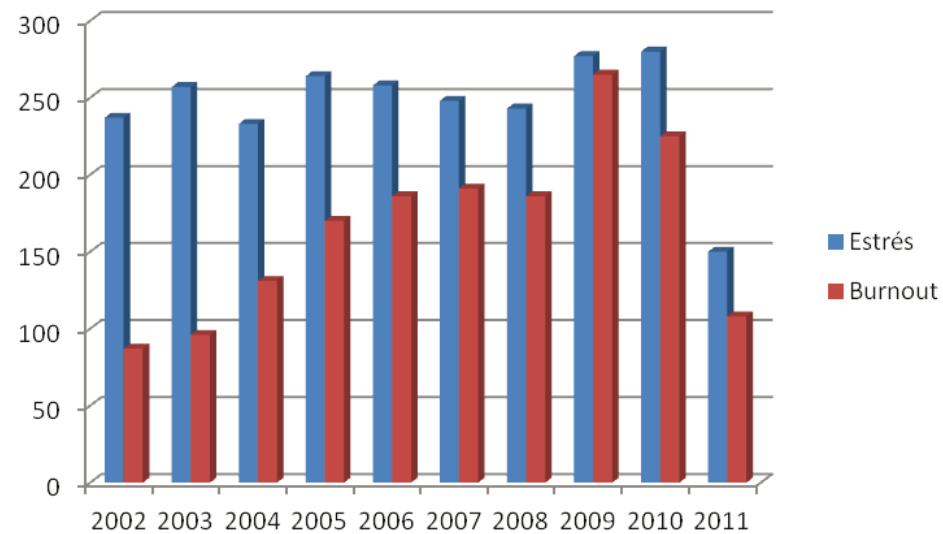


A partir de la revisión de la literatura realizada, el presente artículo tratará de dar una visión global y actualizada del estudio de los procesos de estrés laboral y desgaste profesional, estableciendo los principales modelos y variables de estudio vigentes, junto con las principales líneas de evaluación e intervención que se vienen desarrollando hasta la fecha.

\section{EL ESTRÉS LABORAL Y EL DESGASTE PROFESIONAL: DEFINICIÓN Y ANÁLISIS DEL PROCESO.}

No fue sino fruto del azar y de la observación sistemática, que Hans Selye en la década de los años 30 descubriera lo que en su momento definió como trastorno general de adaptación, hoy conocido como estrés. Selye definió el estrés como una respuesta del organismo ante la percepción de una amenaza caracterizada por una fase de alarma, -donde el organismo se prepara para dar una respuesta-, una fase de resistencia, -donde aparecen los cambios específicos que permitirá enfrentarnos a la situación-, y una fase de agotamiento, -donde se produce un progresivo desgaste de la energía utilizada para hacer frente a la amenaza. Cuando dicha percepción de amenaza se encuentra relacionada con la organización y la gestión del trabajo hablamos entonces de estrés laboral.

El estrés laboral, según la Comisión Europea de Seguridad y Salud en el Trabajo se define como "las nocivas reacciones físicas y emocionales que ocurren cuando las exigencias del trabajo no igualan las capacidades, los recursos o las necesidades del trabajador". En general, y atendiendo al modelo de Karasek ${ }^{4}$ y de Karasek y Theorel ${ }^{5}$, el equilibrio entre la demanda y el nivel de control producirá el grado de estrés en un momento determinado. La ausencia de un proceso de recuperación tras un periodo de estrés continuado, así como la propia naturaleza acumulativa del mismo puede llevar a un estrés crónico. Lazarus y Folkman ${ }^{6}$ definieron el estrés crónico como "una relación particular que se establece entre el sujeto y el entorno que es evaluada por éste como amenazante y desbordante". Y es esta segunda faceta, cuando la persona se percibe totalmente agotada, exhausta, desbordada, como consecuencia de un proceso de desgaste, la que describe el síndrome de burnout o desgaste profesional. Hoy por hoy se utiliza la palabra desgaste profesional como adaptación al español del significado de la palabra burnout, ya que el mismo término de desgaste lleva implícito la referencia a este proceso disfuncional de deterioro ${ }^{7}$. En este sentido, el desgaste profesional hace también referencia al proceso de pérdida lenta, pero considerable, del compromiso y la implicación personal en la tarea que se realiza. La etiqueta anglosajona de burnout se debe a Herbert Freudenberger ${ }^{8}$, un psicólogo clínico neoyorkino que la empleó para describir a aquellos voluntarios que en un periodo más o menos largo, entre uno y tres años, se encontraban desmotivados y faltos de todo interés por su trabajo. Con posterioridad Christina Maslach dio a conocer este concepto en 1977 en una convención de la APA (Asociación Americana de Psicólogos). Aunque no exista una definición unánimamente aceptada, sí existe consenso al considerar que el síndrome de burnout aparece en el individuo como una respuesta al estrés laboral crónico, que surge al trabajar bajo condiciones difíciles, que se caracteriza por un estado de desgaste físico, emocional y cognitivo, y que tiene consecuencias muy negativas para la persona y para la organización.

Si bien conceptualmente las diferencias son relativamente claras entre estrés crónico, burnout y otros trastornos con síntomas similares como puede ser la depresión, no ha sido hasta muy recientemente cuando se ha podido objetivar de manera clínica estas diferencias, mediante el estudio de la carga alostática. La carga alostática es el deterioro de los sistemas del organismo como consecuencia de la respuesta de estrés ${ }^{9}$. Entre los distintos indicadores clínicos de carga alostática se encuentra el aumento de la tensión arterial sistólica y diastólica, la disminución de la variabilidad de la frecuencia cardíaca, el aumento de los niveles de catecolaminas, cortisol y fibrinógeno, la disminución de los niveles séricos y plasmáticos, y el cociente cintura/cadera. En el estudio realizado por Juster y colaboradores ${ }^{10}$, mediante 15 biomarcadores neuroendocrinos, inmunes, 
metabólicos y cardiovasculares se observó un aumento de la carga alostática asociada al aumento de los síntomas de estrés crónico y burnout pero no de depresión.

Sin embargo, la intervención en estrés laboral y en el desgaste profesional no sólo conlleva la actuación sobre los síntomas, sino sobre los distintos antecedentes o agentes estresantes, moduladores y consecuentes que pueden variar tanto a nivel personal como interpersonal y organizacional. Las distintas dinámicas que se establecen entre cada uno de los componentes dentro del contexto socio-histórico en el que nos encontramos han hecho necesario el estudio del estrés laboral y el desgaste profesional desde un modelo procesual.

Dentro de los agentes estresantes, las condiciones laborales, las relaciones interpersonales, ciertas características de personalidad, el tipo de organización y liderazgo, el contexto social y económico, o ciertas características del diseño de la tarea son algunos de los factores más estudiados hasta el momento ${ }^{11}$. A día de hoy, cada uno de estas facetas se ha estudiado por separado, permitiendo una mayor compresión de cada una de ellas. En este sentido, la sobrecarga de trabajo, la ausencia de descansos, largas horas de trabajo, tareas rutinarias con escaso significado y la no utilización de las habilidades del trabajador son algunas de las características del diseño de la tarea que mayores niveles de estrés generan en el trabajador ${ }^{12-13}$. En cuanto a la supervisión, destacan la baja participación en la toma de decisiones, escasos niveles de comunicación y apoyo, junto con la ausencia de políticas de conciliación ${ }^{14}$. Asimismo, el conflicto y la ambigüedad de roles, el exceso de responsabilidad, la escasez de formación y reciclaje, la inseguridad laboral, la ausencia de oportunidades de promoción profesional y desarrollo, son algunas de las características organizacionales y del contexto socio-económico más relevantes ${ }^{15,16}$. Y por último, y no por ello menos importante, las condiciones físicas y ergonómicas del puesto como la contaminación acústica o una ventilación e iluminación inadecuadas ${ }^{11}$.

Revisando los desarrollos más actuales que se desmarcan de estas líneas cabe destacar el estudio realizado en 63 países con una muestra de más de 420.000 participantes en los que se observa los niveles de autonomía y de libertad de elección como el factor más consistentemente relacionado con altos niveles de bienestar y menores niveles de estrés laboral y burnout ${ }^{17}$. Estos datos apoyarían la llamada paradoja Easterlin ${ }^{18}$ donde mayores niveles de ingresos y de estado del bienestar no predicen de manera linear los niveles de bienestar, o inversamente proporcional los niveles de estrés, sino más bien que la relación sería curvilínea, por lo que existiría un punto de saturación donde un mayor poder adquisitivo no conllevaría un aumento de los niveles de bienestar. Sin embargo, según este estudio mayores niveles de autonomía y de libertad de elección sí estarían relacionados con un incremento en los niveles de bienestar, y menores niveles de desgaste profesional.

Entre las principales variables moduladoras que median el efecto de los distintos estresores en la respuesta de estrés laboral y de sintomatología de burnout, clásicamente se encuentra las estrategias de afrontamiento activo y de apoyo social ${ }^{19,20}$, el locus de control $^{21,22}$ la personalidad resistente ${ }^{23,24}$, la estabilidad emocional ${ }^{25,22}$, el sentido de la coherencia $^{26}$, la autoeficacia ${ }^{27,28}$, el patrón tipo A de conducta $^{29}$ y más recientemente el patrón tipo $\mathrm{D}^{20}$, la inteligencia y competencia emocional ${ }^{30,31,24}$, el trabajo emocional y la disonancia emocional ${ }^{32-34}$, o la motivación intrínseca ${ }^{35}$.

La actualización de las metodologías de investigación ha permitido el estudio del impacto longitudinal de algunas de estas variables. En este sentido se ha podido observar como el aumento del uso de estrategias sociales optimistas en detrimento de estrategias de evitación se encontraba asociado con menores niveles de burnout y mayores niveles de engagement a lo largo de los primeros 18 años de experiencia profesional ${ }^{36}$. Asimismo, el apoyo social sigue siendo uno de los factores de mayor peso en la prevención del estrés laboral y el desgaste profesional en los estudios tanto longitudinales ${ }^{37}$ como de meta-análisis ${ }^{38}$. Por otra parte, en los últimos años se ha detectado un posible efecto moderador de variables menos clásicamente estudiadas, entre las que destacarían los 
niveles de religiosidad, la práctica de la meditación, la relajación, el sentido del humor y la creatividad ${ }^{39-43}$, o a nivel social, la eficacia organizacional ${ }^{44}$.

En cuanto a las consecuencias, los procesos de estrés y desgaste profesional se han caracterizado por su impacto global, en la medida en que se observan efectos directos no sólo a nivel individual, sino social y organizacional, siendo la gravedad de las consecuencias significativamente mayores en el caso del burnout. Los efectos encontrados clásicamente mediante metodología transversal sobre los niveles de satisfacción laboral, también han sido hallados en estudios longitudinales tanto como consecuencia del estrés laboral ${ }^{45}$ como del desgaste profesional ${ }^{46}$. También se han detectado consecuencias en cuanto al rendimiento laboral inmediato, muchas veces moderado por factores personales como la inteligencia emocional ${ }^{47}$, o la autoeficacia ${ }^{48}$. En cuanto a la relación entre estrés laboral y desgaste profesional, se ha observado tanto a nivel transversal, como longitudinal y en estudios de meta-análisis ${ }^{49}$ que la propia respuesta de estrés actúa como mediador entre las demandas laborales y la experiencia de desgaste profesional. Por su parte, entre las principales consecuencias del burnout destacan los problemas de salud en general y específicamente problemas psicosomáticos a nivel personal ${ }^{13}$.

Sin embargo, no ha sido hasta los últimos diez años que se han empezado a estudiar factores de riesgo fisiológico e incluso marcadores físicos como medidas objetivas del deterioro por estrés laboral y burnout ${ }^{50}$, tanto en estudios transversales ${ }^{51}$, como longitudinales $^{52}$. En estos estudios se ha observado como mayores niveles de estrés laboral se encuentran asociados con mayores niveles de los principales metabolitos de la noradrenalina en plasma (BDNF y MHPG) ${ }^{51}$, junto con un mayor riesgo de apoplejía principalmente en hombres trabajadores en el sector industrial tras un estudio longitudinal de 11 años $^{53}$, y un aumento del riesgo de trastornos cardiovasculares en general ${ }^{54}$. En el caso del desgaste profesional mayores niveles de burnout se asocian con una mayor carga alostática ${ }^{10}$. Sin embargo, un reciente estudio de meta-análisis realizado por DanhofPont ${ }^{55}$ y colaboradores, indica que muy probablemente, debido a las grandes diferencias en los métodos utilizados hasta el momento para evaluar tanto los biomarcadores como los niveles de desgaste profesional, aun no se ha detectado de manera consistente un perfil específico de factores fisiológicos asociados al burnout. Por último, la inclusión de nuevas metodologías de estudio más acordes con un enfoque procesual están aportando una serie de datos que parecen en principio contradictorios con los obtenidos de manera consistente con metodología transversal. Tal es el caso de los dolores muscoloesqueléticos. En un estudio realizado por Larsman y colaboradores ${ }^{52}$ con una muestra de 1.133 personas evaluadas entre 2004 y 2008 los datos indican que no existiría relación entre padecer altas demandas laborales, estrés laboral y el desarrollo de dolor muscoloesquelético. A pesar del posible impacto de dichos datos, es importante ser cautos y esperar a que nuevos estudios de corte longitudinal lo confirmen.

Por otra parte, a nivel social, las principales consecuencias vienen asociadas a las actitudes y conductas de carácter negativo desarrolladas por el sujeto, como consecuencias del estrés y el desgaste profesional, tales como la suspicacia, la agresividad, el aislamiento, o la irritabilidad. Estas actitudes pueden deteriorar ostensiblemente las relaciones interpersonales generando una serie de consecuencias en el estrés laboral y el desgaste profesional como son los conflictos interpersonales, la evitación de contactos sociales, o la pérdida de redes de apoyo ${ }^{56,13}$. Asimismo, a través de metodologías de diario y experimentales se ha podido comprobar los efectos crossover o de contagio tanto en estrés laboral ${ }^{57}$ como en desgaste profesional $^{58}$. Los mecanismos del proceso se han explicado hasta la fecha mediante procesos de similitud con la persona afectada ${ }^{59}$ o mediante procesos de disonancia emocional y falta de autenticidad en las relaciones interpersonales ${ }^{60}$.

A nivel organizacional, entre las principales consecuencias tanto del estrés laboral como del desgaste profesional destacan el abandono o intenciones de abandono del puesto de trabajo, el absentismo laboral, y un mayor número de rotación y bajas laborales ${ }^{61,62}$. De manera relativamente reciente se han podido confirmar estos efectos también mediante metodología longitudinal y estudios de meta-análisis. Tal es el caso del absentismo laboral 
en una muestra de 844 trabajadores a lo largo de un año, donde se observó que el incremento en los niveles de burnout producía un aumento de la tasa de absentismo un año después ${ }^{46}$. Asimismo, diversos estudios de meta-análisis han mostrado un tamaño del efecto significativo en la relación entre estrés laboral, desgaste profesional, intenciones de abandono y abandono del puesto ${ }^{49}$. También se han estudiado los efectos del estrés laboral y el burnout en temas de seguridad laboral. Los datos recogidos hasta el momento en un reciente estudio de meta-análisis con más de 200 muestras independientes informan de una relación positiva y un tamaño del efecto significativo en la relación entre el desgaste profesional y las conductas de riesgo y el número de accidentes de trabajo ${ }^{63}$. Por último, metodologías multinivel han permitido demostrar cómo un aumento del estrés laboral a nivel individual, con una muestra de 924 trabajadores, se relaciona significativamente con un decremento de producción a nivel organizacional ${ }^{48}$.

Si bien estas son algunas de las características que de manera general se ha observado en distintos grupos profesionales, ya en 1993, Schaufeli, Maslach y Marek ${ }^{64}$ afirmaban que el estudio de la etiología del estrés laboral y el desgaste profesional debe atender a la propia naturaleza del trabajo y de los estresores asociados, por lo que es conveniente la investigación específica de cada entorno laboral relativo a cada profesión. Y es esta perspectiva de especificidad la que ha ido marcando las distintas líneas de estudio y desarrollo en ambos campos de investigación hasta la fecha. A nivel general, cabe destacar que el sector servicios sigue siendo el más estudiado, y concretamente el profesorado con más de 2000 artículos, seguido de enfermería, cuerpo de policía, y médicos con un total de 506 artículos. El número de estudios realizados en el resto de profesiones ya se encontraría por debajo de los 200 artículos aproximadamente.

\section{MODELOS DE ESTUDIO EN LOS PROCESOS DE ESTRÉS LABORAL Y DESGASTE PROFESIONAL}

Todos estos datos nos indican, que si bien hemos avanzado notablemente en el estudio de cada uno de los componentes del proceso, aún no se conoce en profundidad la dinámica de relación de estos factores que degenera en la experiencia de estrés de manera diferencial en los profesionales. A continuación vamos a tratar de desarrollar por orden de relevancia -atendiendo al número de publicaciones donde se ha comprobado empíricamente el modelo-, las distintas teorías explicativas del estrés laboral y del desgaste profesional.

\section{Modelos de proceso de estrés laboral}

\section{Modelo de Ajuste Persona-Ambiente de Harrinson ${ }^{65}$}

La distinción entre persona y ambiente (P-A) es una de las características básicas en este modelo. Esta distinción se convierte por tanto en un pre-requisito para la conceptualización de los niveles de ajuste P-A, siendo esta la base de causación recíproca entre el individuo y su entorno. La segunda característica del modelo es la distinción explícita entre dimensiones "objetivas" y perceptivas tanto de la persona como del ambiente. En este sentido, Harrinson considera importante analizar el efecto diferencial entre características objetivas tanto de la persona y el ambiente frente a percepciones del individuo tanto de sí mismo (i.e. autoconcepto) como del entorno. Por tanto, al establecer los niveles de ajuste, se analizan tanto los niveles de ajuste persona-ambiente, objetivo y perceptivo, así como los niveles de ajuste entre la percepción del ambiente y sus características objetivables, y el nivel de precisión en la autovaloración del sujeto. La aplicación de este modelo en las distintas investigaciones empíricas ha llevado a una modificación del mismo al observar que los niveles de ajuste objetivos persona-ambiente no mostraban un impacto apreciable en la salud mental, a menos que fuese percibido por el sujeto y por tanto llevado a la dimensión subjetiva de los niveles de ajuste ${ }^{6}$. 


\section{Modelo de facetas ampliado de Beehr y Newman ${ }^{66}$}

En este modelo (ver figura 2), el estrés laboral se localiza en la faceta medioambiental, siendo la respuesta de estrés parte de la faceta de consecuencias personales. Según el modelo la presencia de estresores y de respuesta de estrés serían las dos condiciones necesarias y suficientes para hablar de una situación de estrés ocupacional. La presencia de otras facetas, indicarían el posible efecto de otras variables en el proceso. La faceta personal se compone de características relativamente estables de la persona (por ejemplo, la personalidad, sus rasgos o características socio-demográficas). Éstas podrían interactuar con distintos estresores para producir una variabilidad más amplia en la respuesta de estrés. La variable consecuencias organizativas contiene los comportamientos de los empleados, en situaciones de estrés, que tienen implicaciones directas en la eficacia de la organización (por ejemplo, el absentismo, la rotación o cambios en los niveles de rendimiento). Por último, la faceta duración reconoce la importancia del tiempo en el propio proceso de estrés laboral, que es probablemente un factor poco reconocido en el estudio del proceso ${ }^{67}$. Posteriormente, se desarrolló un modelo extendido en el que se rompe la faceta ambiental en dos partes, estresores laborales y otras características de la situación. Estas otras características de la situación podrían moderar la relación entre los estresores y el resto de las variables del proceso. Además, en esta nueva versión del modelo, cambiaría el papel de las características personales para mostrar de forma explícita su papel moderador en la relación estresores y respuesta de estrés. En este sentido, las características personales podrían también dar lugar directamente a distintos tipos de respuesta de estrés. Asimismo, "el afrontamiento y la adaptación" aparecen en este modelo para indicar las medidas adoptadas para corregir los problemas derivados de los estresores, en las respuestas de estrés y los efectos a nivel organizacional.

Figura 2. Modelo de facetas ampliado de Beehr y Newman ${ }^{66}$

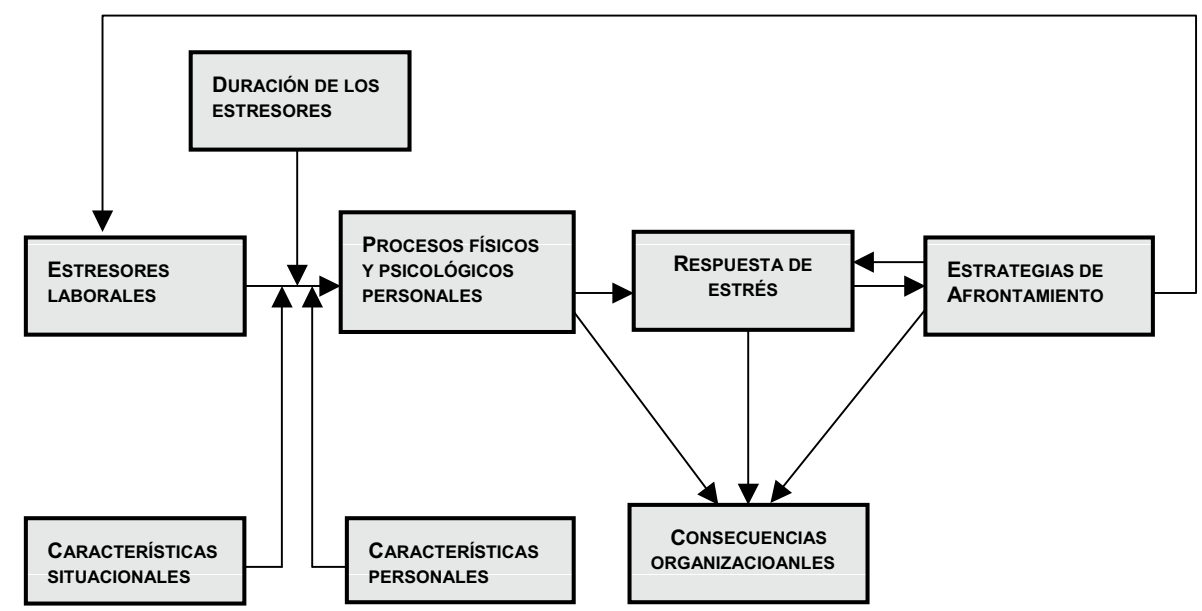

\section{Modelo Cibernético del estrés laboral de Edwards ${ }^{68}$}

Este modelo trata de integrar aspectos de la literatura sobre auto-regulación y el estrés ocupacional. De acuerdo con este modelo, el estrés en las organizaciones podría ser conceptualizado no en términos de las distintas características de la persona o del ambiente sino en términos de la relación entre ambos dos. En este sentido, el estrés organizacional se define como los niveles de discrepancia entre la percepción del estado actual en el que se encuentra la persona y el estado deseado, en la medida en que el sujeto considera importante esta discrepancia ${ }^{68}$. Las concepciones del estado tanto actual como deseado se describirían de manera amplia, abarcando tanto la representación subjetiva de las condiciones laborales actuales como las metas, valores y objetivos específicos que la persona desea alcanzar. Por tanto, la formulación y operativización empírica del modelo requeriría el uso de una metodología de establecimiento de metas y objetivos específicos de cada persona evaluada, como la desarrollada por Emmos ${ }^{69}$, lo que da una validez ecológica amplia al uso de este paradigma. Sin embargo, el número 
de estudios empíricos desde esta perspectiva, si bien han mostrado un valor añadido significativo, son bastante reducidos ${ }^{70}$.

\section{Teoría de la Activación Cognitiva del Estrés de Meurs ${ }^{71}$}

Nace recientemente como teoría explicativa del estrés laboral que pretende integrar los modelos existentes hasta la fecha recogiendo desarrollos realizados en el estudio del estrés ajenos al entorno organizacional ${ }^{72}$. El modelo original establece que es necesario analizar cuatro niveles distintos de estrés. Por un lado, el estrés como estímulo, los estresores, que toman ese rol en la medida en que el sujeto percibe el estímulo y lo evalúa como amenaza. Dicha evaluación dependería de las experiencias previas y las expectativas de resultado que tenga el propio sujeto. En segundo lugar, la experiencia de estrés, es decir, la percepción subjetiva cognitiva y emocional de sentirse estresado. Posteriormente la respuesta de estrés, entendida desde este paradigma como el incremento de arousal cerebral o como proceso de activación por estrés. En este sentido, la evaluación de la respuesta de estrés se realiza mediante registros psicofisiológicos, neuroendocrinos, psicoinmunológicos, comportamentales y de bioquímica cerebral. Y por último, el feedback de la respuesta de estrés, donde la persona percibe los cambios periféricos producidos por la respuesta de estrés, o lo que es lo mismo, la propia experiencia de la respuesta de estrés. Debido a la novedad de la inclusión de este paradigma en el estudio del estrés laboral, aún no constan estudios empíricos en las bases de datos.

\section{Modelos de proceso de Desgaste Profesional}

\section{Modelo de Demandas y Recursos de Demerouti y colaboradores ${ }^{73}$}

El modelo de Demandas y Recusos laborales (ver figura 3) enfatiza principalmente la existencia de dos líneas paralelas de desarrollo, por un lado las demandas laborales y el desgaste o agotamiento del profesional, y por otro los recursos laborales y la desimplicación. Este modelo asume que cuando las personas se encuentran en una situación donde las demandas laborales son altas y los recursos limitados, esto producirá una situación de desgaste energético y de desmotivación que favorecería el desarrollo de desgaste profesional, sea cual sea el tipo de profesión. Las demandas laborales se refieren a aquellos aspectos físicos, sociales y organizacionales que requieren un esfuerzo físico o mental mantenido y que por tanto conlleva un coste psicológico y fisiológico. Basado en el modelo de control de la demanda de gestión de Hockey ${ }^{74}$, las demandas laborales se perciben como estresores ambientales donde las personas tienen que desarrollar estrategias de protección frente a ellos. Por otro lado, los recursos laborales hacen referencia a aspectos tanto físicos, como psicológicos, sociales u organizacionales que facilitan la consecución de los objetivos laborales y reducen los costes físicos y psicológicos asociados a las demandas, estimulando el desarrollo y crecimiento personal. Por tanto el proceso de desgaste seguiría dos caminos. El primero, relacionado con el agotamiento y la extenuación producidos por la sobrecarga de las demandas laborales, y el segundo vinculado con la falta de recursos que exacerba los efectos negativos de las demandas y que degenera en conductas de desimplicación y abandono. Las últimas conceptualizaciones de este modelo $^{75}$ reducen significativamente el número de demandas laborales a factores de sobrecarga, y también en el número de recursos permaneciendo el apoyo social. Asimismo, aparecería una variable de recursos personales relacionada con aquellos aspectos de la persona que se encuentran generalmente relacionados con factores de resiliencia. Es decir, las percepciones de las personas en cuanto a su capacidad para controlar y actuar con éxito sobre su entorno. Estos recursos actuarían como moduladores del proceso. 
Figura 3. Modelo extendido sobre demandas y recursos laborales ${ }^{73}$

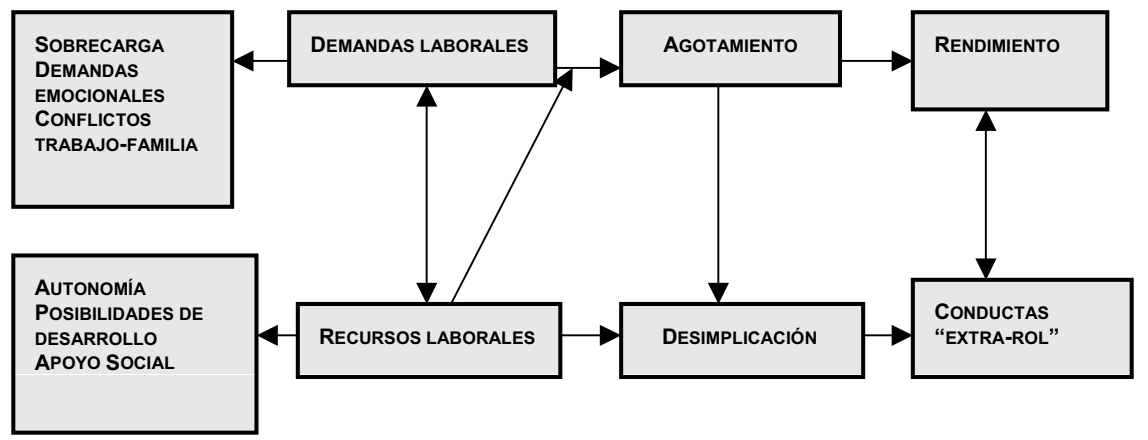

\section{Modelo de Conservación de Recursos de Hobfoll76}

Esta teoría explicativa del desgaste profesional se centra principalmente en aquellos factores cognitivos y del ambiente asociados con los recursos personales, concebidos como "objetos, características personales, condiciones o energías que son valiosos por sí mismos, o porque proveen o protegen recursos valiosos para la persona" 77 (ver figura 4). Por tanto, los recursos se convierten en el factor central de este modelo. El principio básico de esta teoría afirma que las personas intentan adquirir, mantener y proteger los recursos que poseen, percibiendo la pérdida potencial o real de recursos, así como la pérdida de una ganancia esperada como estresante. Por tanto, la pérdida de estos recursos o la mera amenaza de dicha pérdida se convertiría en agente causante de la experiencia de estrés. En este sentido, el desgaste profesional o burnout se concibe como el resultado de esa pérdida global o amenaza de pérdida de recursos valiosos, y por tanto, como un síndrome fundamental de agotamiento de recursos. Además, Hobfoll ${ }^{76}$ sugiere que las características personales podrían actuar como moduladores frente a la experiencia de estrés. Asimismo, las relaciones sociales desde este modelo son vistas como recursos, en la medida en que son proveedores o facilitadores de la preservación de recursos valiosos para el sujeto.

Figura 4. Modelo teórico sobre la conservación de los recursos ${ }^{77}$

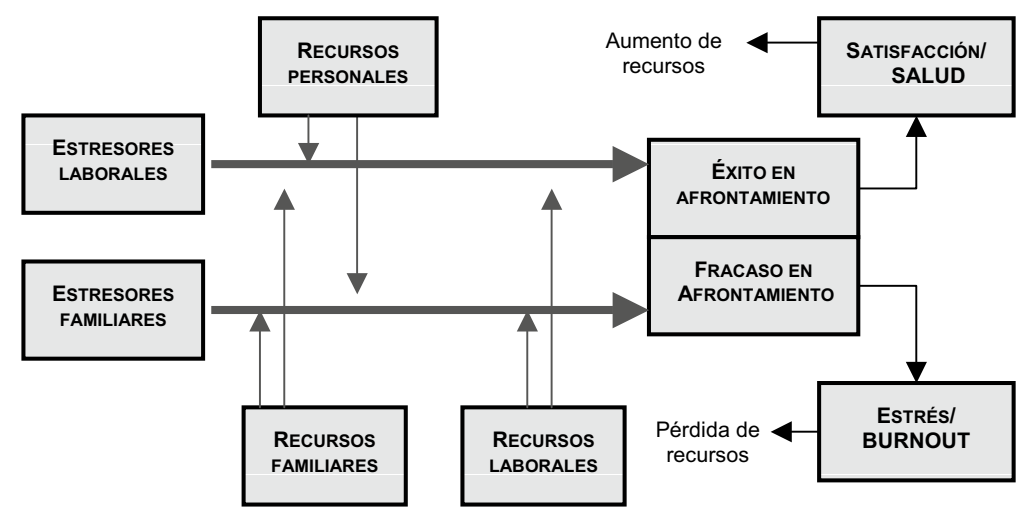

\section{Modelo de Esfuerzo-Recompensa de Siegrist ${ }^{78}$}

El modelo de esfuerzo-recompensa enfatiza la importancia de los roles sociales en la medida en que pueden ofrecer a la persona oportunidades de contribuir o realizar tareas (generando sensaciones de auto-eficacia), oportunidades de sentirse recompensados y valorados (incrementando la autoestima) y oportunidades de pertenencia a un grupo significativo para la persona. A partir de estas consideraciones, esta teoría establece la falta de reciprocidad o equidad entre ganancias y costes como agente causante de un estado de distrés emocional que degeneraría en desgaste profesional y problemas de salud. Asimismo, el modelo asume que en muchos trabajos no existe una completa especificación del puesto que favorezca un balance equilibrado entre los esfuerzos requeridos y las recompensas que se otorgan. Estos sentimientos de ser tratado 
injustamente y de falta de reconocimiento como resultado de un sistema inapropiado de recompensas generan una serie de respuestas de estrés del sistema nervioso autónomo. Este hecho es debido a la experiencia recurrente de déficit de recompensa que impediría la capacidad de regulación y adaptación al entorno. A largo plazo, este nivel de desajuste entre altos niveles de esfuerzo y baja recompensa conduciría a un desgaste profesional y aumentaría el riesgo de enfermedad como consecuencia del estrés mantenido.

\section{Modelo Transaccional de Moreno-Jiménez ${ }^{79}$}

Las investigaciones clásicas en estrés organizacional y desgaste profesional generalmente se han centrado en los efectos directos de las variables organizacionales, sociales, de la tarea o variables de personalidad como agentes causantes e independientes del proceso de estrés. Sin embargo, si seguimos el modelo teórico cognitivo-motivacionalrelacional de Lazarus $^{80}$, esta asunción entra en conflicto con una concepción de las respuestas emocionales como dependientes de los significados relacionales construidos como fruto de la interacción entre la persona y el ambiente. De hecho, Lazarus ${ }^{80}$ considera ineficaz como medida de reducción del estrés, intentar modificar las condiciones laborales sin tener en cuenta los objetivos, metas, recursos y creencias de los trabajadores. De hecho, muchos estudios han tratado de reflejar esta variabilidad interindividual en la percepción de las variables organizacionales, si bien en muchos casos se ha quedado reducido a un tratamiento estadístico de los datos sin tener un reflejo real por evaluar la manera en que las experiencias laborales son percibidas en interpretadas por la persona. Desde esta perspectiva, el equipo de Moreno-Jiménez viene desarrollando desde sus inicios una perspectiva transaccional en el estudio de distintos procesos socio-laborales, como son el desgaste profesional, el estrés traumático secundario, el acoso laboral o los procesos de conciliación familiar-laboral, donde las variables de personalidad interactúan a lo largo de la generación, desarrollo y consecuencia de los mismos (ver figura 5).

Figura 5. Modelo procesual y transaccional de Desgaste Profesional 79

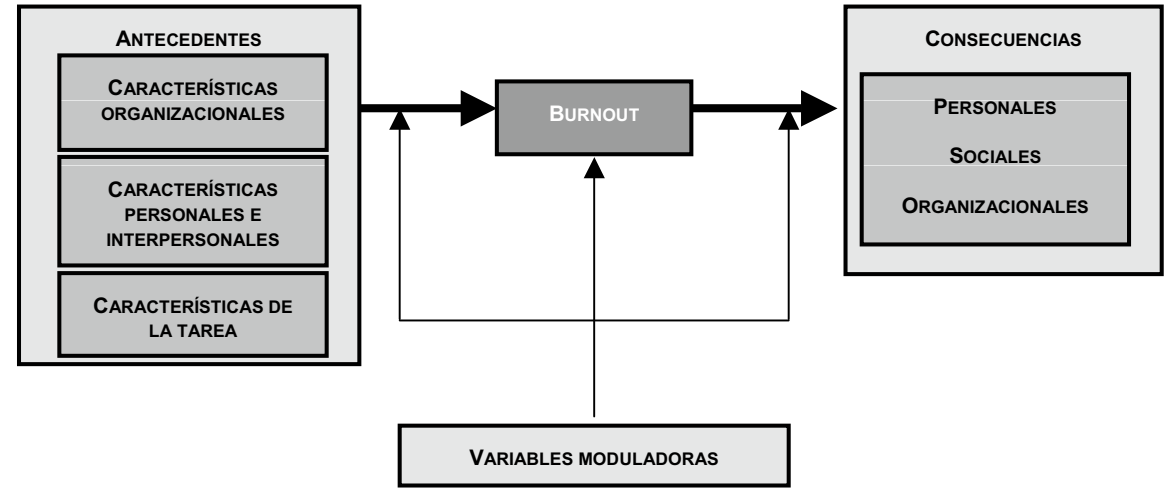

\section{Modelo de interacción entre Individuo y Organización de Leiter y Maslach ${ }^{12}$}

A través de un conjunto de datos normativos, con más de 8.000 participantes, que cubren una amplia gama de ocupaciones a través de Canadá, Estados Unidos, Europa y Asia, Michael Leiter y Christina Maslach ${ }^{12}$ han validado la escala de vida laboral, un cuestionario que trata de recoger algunas variables individuales y organizativas identificadas por los autores como las áreas más relevantes del entorno de trabajo. El modelo teórico que Leiter y Maslach ${ }^{12}$ elaboraron para el desarrollo de esta considera que un desajuste entre las personas y su entorno de trabajo en estas áreas, reduce la capacidad de energía, implicación y sensación de eficacia, mientras que el ajuste produciría el efecto contrario, aumentando los niveles de compromiso. Una de las áreas más importantes considerada por los autores y la literatura científica es la carga de trabajo, que se define como la cantidad de trabajo que se realiza en un momento dado. Los autores afirman que un volumen de trabajo asequible ofrece la oportunidad de disfrutar con la tarea, para conseguir los objetivos laborales, y su desarrollo profesional. Sin embargo, el exceso de trabajo no se conceptualizaría simplemente como un desfase o exceso en los requisitos 
para conseguir un nuevo reto, sino ir más allá de los límites humanos. El control se considera en este modelo como el segundo factor más importante y se define como la oportunidad de hacer elecciones y tomar decisiones, resolver problemas, y contribuir al cumplimiento de las responsabilidades. Un buen ajuste se produce cuando hay una correspondencia entre el control y los niveles de responsabilidad, produciéndose una discrepancia cuando las personas carecen de control suficiente para cumplir con las tareas de las que se les hace responsables. La recompensa sería un tercer factor que recogería tanto el reconocimiento económico como social del trabajo, ofreciendo indicadores claros de lo que valora la organización, mientras que la falta de reconocimiento devalúa tanto el trabajo como a ellos mismos. El factor de comunidad se define como la calidad del entorno social de una organización relacionado con el apoyo social, la colaboración y los sentimientos positivos. Los desajustes se producen cuando no existe una conexión positiva con los demás en el trabajo. La equidad considerada como la medida en que la organización tiene normas coherentes y equitativas para todos. Un elemento importante en este factor es el grado en que los recursos se asignan de acuerdo a procedimientos comprensibles y consistentes. La equidad transmite respeto por los miembros de la comunidad. La falta de equidad indica confusión en los valores de una organización y en sus relaciones con las personas. Finalmente, los autores consideran los valores como lo que la organización y sus miembros consideran importante. En este sentido, cuando los valores organizacionales y personales son congruentes, los éxitos son compartidos, por su parte, cuando existen diferencias entre los valores de una organización y los valores de su personal, o si la organización no practica sus valores se producen desajustes que degeneran en estrés y desgaste.

\section{Burnout como un proceso de desilusión en la profesión de ayuda de Edelwich y Brodsky ${ }^{81}$}

La mayoría de las personas en el inicio de su profesión experimentan lo que podríamos llamar una fase de entusiasmo por el acceso a un nuevo puesto de trabajo, con nuevas expectativas e intereses. Durante algún tiempo todo contratiempo se contrarresta con las energías que proporciona la motivación por hacer un "buen trabajo". Si las dificultades y la realidad del día a día empiezan a romper expectativas pasamos a una fase de estancamiento donde empezamos a plantearnos si el nivel de esfuerzo personal compensa los beneficios. Una vez que empezamos a plantearnos la posible eficacia del esfuerzo personal realizado para superar los obstáculos laborales, sino disponemos del apoyo de recursos organizacionales, sociales e individuales, nos encontraríamos con una alta probabilidad de pasar a una fase de frustración y posteriormente de apatía y pérdida de referencias, que desencadenaría en el síndrome de desgaste profesional.

\section{LÍNEAS ACTUALES DE INTERVENCIÓN}

Los altos costes personales, sociales y organizacionales generados por el estrés laboral y el desgaste profesional, han dado lugar a que organizaciones internacionales como la Unión Europea y la OMS insistan cada vez más en la importancia que tiene la prevención e intervención en el ámbito laboral. Paradójicamente, la mayoría de programas se han realizado a nivel individual a pesar de que las condiciones de trabajo y en el desarrollo de ambos procesos. No obstante, las organizaciones comienzan a tomar conciencia de la necesidad de implementar programas tanto de prevención como de intervención. En este sentido, algunos servicios de prevención de riesgos laborales comienzan a desarrollar programas centrados en variables de la propia organización. Este tipo de programas suelen encuadrase bajo la denominación de programas de control de estrés.

Entre los principales procedimientos incluidos a nivel organizacional destacan el rediseño de tareas, la clarificación en los procesos de toma de decisiones, mejora de la supervisión, establecimiento de objetivos y procedimientos específicos y delimitados 
para cada unos de los roles profesionales, líneas claras de autoridad, mejora de las vías de comunicación dentro de la organización, mejora de las condiciones físicas y ergonómicas de trabajo, mejora de los sistemas de incentivos y recompensas, ajuste de la promoción de los profesionales siguiendo criterios claros, objetivos y equitativos, mejoras en el ajuste de los niveles de autonomía y responsabilidad del empleado, flexibilidad laboral y políticas de conciliación, etc.

La percepción del apoyo social es crucial para el individuo. Tal y como comentábamos al principio de este artículo, si tenemos en cuenta que pasamos una gran parte de nuestro tiempo en el trabajo, las relaciones interpersonales con los compañeros y clientes o usuarios cobran una especial relevancia para nuestra identidad como personas y nuestro equilibrio físico y mental. A través de estas redes sociales de apoyo las personas obtienen información, adquieren nuevas habilidades o mejoran las que ya poseen, obtienen refuerzo social, apoyo emocional y feedback sobre el nivel de ejecución de la tarea. Aquellos programas, tanto de prevención como de intervención, que incluyan este tipo de estrategias permitirán mejorar de manera casi inmediata el clima de trabajo. Entre las estrategias para el aumento de la calidad de las relaciones entre los trabajadores destacan la creación de grupos de apoyo, creación de grupos de trabajo, entrenamiento en habilidades sociales, entrenamiento en manejo de situaciones conflictivas, habilidades comunicativas...

Asimismo, las estrategias individuales orientadas al manejo del estrés o el aprendizaje de técnicas de relajación puede ayudar a manejar el agotamiento y el desgaste psíquico que generan situaciones de estrés agudo y estrés crónico, si bien este tipo de programas son relativamente poco eficaces en el lugar de trabajo, ya que la persona tiene menos control sobre las fuentes de estrés. Asimismo, el ejercicio físico reduce los niveles de ansiedad de manera significativa pero no impide el desarrollo del desgaste profesional. En el ámbito de la prevención del desgaste profesional a nivel individual se recomiendan otros tipos de programas entre los que destacan el entrenamiento en resolución de problemas, entrenamiento para el manejo eficaz del tiempo, y la adopción de estilos de vida saludables que faciliten el distanciamiento del trabajo en horario extra-laboral, entre otros. Entre los programas comentados cabe destacar el entrenamiento en solución de problemas. Esta técnica pretende ayudar a las personas a resolver problemas y mejorar su toma de decisiones. Para ello se le entrena en la identificación y reconocimiento del problema, detección de las diferentes alternativas, elección de la respuesta más adecuada y verificación de su idoneidad.

Cabe destacar que las líneas de desarrollo más actuales en cuanto a los programas de control y manejo del estrés y el desgaste profesional se orientan principalmente al uso de nuevas tecnologías para elevar los índices de penetración en el mercado laboral y de adhesión por parte de los participantes. En este sentido, recientemente se han publicado algunos datos empíricos sobre los efectos de este tipo de intervención con soporte web ${ }^{82}$ o de apoyo mediante el uso de foros online ${ }^{83}$.

Las terapias basadas en la aceptación y el contacto con el momento presente (por ejemplo: Terapia de Aceptación y Compromiso y Mindfulness o Conciencia Plena) están poco a poco acumulando evidencia a favor de la utilización de las mismas en el ámbito laboral con el objetivo de prevenir y/o reducir el estrés y otros efectos negativos del entorno laboral ${ }^{84}$. La terapia de aceptación y compromiso (ACT) busca aumentar la flexibilidad psicológica del individuo. La flexibilidad psicológica se define como estar en contacto con el momento presente, como un ser humano consciente y, en base a lo que la situación ofrece, de acuerdo con unos valores elegidos ${ }^{85}$. Durante la intervención se trabajan seis componentes de la misma: la aceptación, la defusión cognitiva, el contacto con el momento presente, el yo como contexto, el esclarecimiento de los valores del individuo y la acción comprometida. Cada uno de estos procesos no son solamente una estrategia para prevenir y aliviar el estrés o el burnout, sino que son habilidades psicológicas positivas en sí mismas ${ }^{84}$. Por otra parte las terapias basadas en mindfulness buscan que el individuo mejore sus capacidades de concentración en el momento 
presente, focalizando su energía y su atención, sin juzgar ${ }^{86}$. Ambas comparten elementos comunes, es por ello que se suele hacer referencia a ellas de manera conjunta ${ }^{87}$.

Diversos estudios de intervención en el ámbito laboral han demostrado que ACT resulta eficaz en el entorno laboral. En general la investigación en el área ha encontrado que mayores niveles de flexibilidad psicológica y/o el aumento de la misma a través de las intervenciones, se relaciona con resultados positivos para los empleados y por ende para las empresas ${ }^{88}$. Por ejemplo, ACT se ha mostrado eficaz en la ayuda a los cuidadores profesionales (trabajadores sociales, policías, médicos) a la hora de enfrentarse con el peligro y el sufrimiento humano, así como para reducir la incidencia del trauma secundario $\mathrm{o}$ indirecto ${ }^{89}$. Igualmente se he encontrado que es eficaz tanto en la prevención del desgaste profesional ${ }^{90}$, como en la reducción del mismo ${ }^{91}$ y del estrés laboral ${ }^{91-93}$; y que a pesar de que las características del propio lugar de trabajo son importantes en los programas de intervención tanto del estrés laboral como del desgaste profesional, los procesos basados en la aceptación, los valores y el mindfulness parecen mostrar una relación más fuerte y consistente en la reducción de ambos ${ }^{87}$.

Una última línea de intervención a destacar busca aumentar la resiliencia de los empleados a través de entrenamientos enfocados en hacer "más fuertes" a los individuos ante el estrés, de tal forma que éste no llegue a manifestarse como tal. Serían programas principalmente enfocados al desarrollo de estrategias de resistencia, resiliencia y recuperación ${ }^{94}$. Sin embargo, a partir de la revisión realizada, no existen aún estudios empíricos que muestren su eficacia y su diferencia con otros programas de intervención. Sin embargo, sí existen datos empíricos en programas específicos centrados en el entrenamiento de estrategias de recuperación ${ }^{95}$ que han demostrado ser eficaces en la reducción del estrés percibido y el afecto negativo, así como la mejora de la calidad del sueño.

\section{REFERENCIAS BIBLIOGRÁFICAS}

1. Domínguez E, Ullíbarri M, Zabaleta I. Reduction of working hours as a policy of work sharing in the face of an economic crisis. Applied Economics Letters. 2011; 18(7): 683.

2. Turner, N., Chmiel, N., Sandy Hershcovis, M., Walls, M. Life on the line: Job demands, perceived co-worker support for safety, and hazardous work events. J Occup Health Psychol 2010;15 (4): $482-493$.

3. Paton, N. Time to tackle stress at work. Occup Health 2011 Jun; 63 (6) : 17-19.

4. Karasek, R. Job demands, job decision latitude, and mental strain: Implications for job redesign. Adm Sci Q 1979; 24: 285-309.

5. Karasek, R., Theorell, T. Healthy work. Stress, productivity, and the reconstruction of working life. New York: Basic Books; 1990.

6. Lazarus, R.L. y Folkman, S. . Stress, Appraisal, and Coping. New York: Springer; 1984

7. Moreno-Jiménez, B., Oliver, C., Aragoneses, A. . El «burnout», una forma específica de estrés laboral. In: Buela-Casal, G. y Caballo, V.E. Manual de Psicología Clínica Aplicada Madrid: Siglo XXI; 1996 p. $271-284$.

8. Freudenberger, H.J. Staff burnout. J Soc Issues 1974; 30(1): 159-165.

9. McEwen B. Allostasis and Allostatic Load: Implications for Neuropsychopharmacology. Neuropsychopharmacology, 2000; 22:108-124

10. Juster, RP, Sindi, S, Marin, MF, Perna, A, Hashemi, A, Pruessner, JC, Lupien, SJ, et al.. A clinical allostatic load index is associated with burnout symptoms and hypocortisolemic profiles in healthy workers. Psychoneuroendocrinology 2011 Jul; 36 (6): 797-805.

11. Cooper CL. Theories of organizational stress. New York: Oxford University Press, Inc; 1998.

12. Leiter, MP, Maslach, C. Areas of worklife: A structured approach to organizational predictors of job burnout. In Perrewe PL, Ganster DC, editors. Research in occupational stress and well being: Vol. 3. Emotional and physiological processes and positive intervention strategies. Oxford, UK: JAI Press/ Elsevier.; 2004. p. 91-134.

13. Schaufeli WB, Enzmann D. The burnout companion to study and practice: A critical analysis. London: Taylor y Francis Ltd; 1998. 
14. Taris, TW, Stoffelsen, JM, Bakker, AB, Schuafeli, WB, van Dierendonck D. . Differences in burnout risk between jobs and individuals: About the role of job autonomy. Gedrag \& Gezondheid: Tijdschrift voor Psychologie en Gezondheid 2002; 30(1): 17-29.

15. Collins, VA. A meta-analysis of burnout and occupational stress. Information \& Learning 2000 Mar.

16. Lee RT, Ashforth BE.. On the meaning of Maslach's three dimensions of burnout, J Appl Psychol 1996; 75(6):743-747.

17. Fischer R, Boer D. What is more important for national well-being: Money or autonomy? A meta-analysis of well-being, burnout, and anxiety across 63 societies. J Pers Soc Psychol 2011 Jul; 101(1): 164-184.

18. Easterlin RA. . Diminishing marginal utility of income? Caveat emptor. Soc Indic Res 2005; 70: 243-255.

19. Shinn M, Rosario, M, Mørch, H, Chestnut, DE. Coping with job stress and burnout in the human services. J Pers Soc Psychol 1984 Apr; 46 (4): 864-876.

20. Polman, R,Borkoles, E,Nicholls, AR. Type D personality, stress, and symptoms of burnout: The influence of avoidance coping and social support. Br J Health Psychol 2010 Sep; 15 (3): 681-696.

21. Glass, DC, McKnight, JD.. Perceived control, depressive symptomatology and professional burnout: a review of the evidence. Psyhcology and health 1996; 11: 23-48.

22. De Hoogh, AHB, Den Hartog DN. Neuroticism and locus of control as moderators of the relationships of charismatic and autocratic leadership with burnout. J Appl Psychol 2009 Jul; 94(4): 1058-1067.

23. Kobasa SC. . The hardy personality: toward a social psychology of stress and health. In Sanders GS, Sals J. editors. Social psychology of health and illness . Hillsdales, NJ: Lawrence Erlbaum Associates, Inc; 1982. p. 3-32.

24. Garrosa E, Moreno-Jiménez B, Rodríguez-Muñoz A, Rodríguez-Carvajal R. Role stress and personal resources in nursing: A cross-sectional study of burnout and engagement. Int J Nurs Stud 2011 Apr; 48 (4): 479-489.

25. Hills $\mathrm{H}$, Norvell $\mathrm{N}$. An examination of hardiness and neuroticism as potential moderator of stress outcomes. Behavioural Medicine 1991; 17: 31-38.

26. Carmel S, Anson O, Levenson A, Bonneh DY, Mahoz B. Life events, sense of coherence and health: gender differences on the kibbutz. Soc Sci Med 1991; 32: 1089-1096.

27. Stetz TA, Stetz MC, Bliese PD. The importance of self-efficacy in the moderating effects of social support on stressor-strain relationships. Work Stress 2006; 20(1): 49-59.

28. VanYperen Nico W. Informational support, equity and burnout: The moderating effect of self-efficacy. J Occup Organ Psychol 1998 Mar; 71(1): 29-33.

29. Burke JM. The relationship between the type A behavior, role stress, job enrichment and burnout among college counselors. Disertation Abstracts International; 1985.

30. Weisinger H. Emotional intelligence at work. New York: Jossey-Bass; 1997.

31. Salovey P,Stroud LR, Woolery A, Epel ES. Perceived emotional intelligence, stress reactivity, and symptom reports: Further explorations using the trait meta-mood scale. Psychology \& Health 2002; 17(5): 611-627.

32. Brotherridge CM, Grandey AA. Emotional labor and burnout: Comparing two perspectives of "people work". J Vocat Behav 2002 Feb; 60 (1): 17-39.

33. Moreno-Jiménez B, Gálvez M, Rodríguez-Carvajal R, Garrosa E. Emociones y salud en el trabajo: análisis del constructo "trabajo emocional" y propuesta de evaluación. Rev Latinoam Psicol 2010; 42(1): 63-73.

34. Diestel S, Schmidt KH. The moderating role of cognitive control deficits in the link from emotional dissonance to burnout symptoms and absenteeism. J Occup Health Psychol 2011 Jul; 16 (3): 313-330.

35. Rubino C, Luksyte A, Perry SJ, Volpone SD. How do stressors lead to burnout? The mediating role of motivation. J Occup Health Psychol 2009 Jul; 14 (3): 289-304.

36. Salmela-Aro K, Tolvanen A, Nurmi JE. Social strategies during university studies predict early career work burnout and engagement: 18-year longitudinal study. J Vocat Behav 2011 Aug; 79 (1): 145-157.

37. Sundin L, Hochwälder J, Lisspers J. A longitudinal examination of generic and occupational specific job demands, and work-related social support associated with burnout among nurses in Sweden. Work 2011; 38(4): 389-400.

38. Halbesleben, JRB. Sources of Social Support and Burnout: A Meta-Analytic Test of the Conservation of Resources Model. J Appl Psychol 2006; 91 (5): 1134 -1145

39. Kovács B, Kézdy A. Religious belief and burnout. EJMH 2008 Dec; 3(2): 253-265. 
40. Krasner MS, Epstein RM, Beckman H, Suchman AL, .; Chapman NB, Mooney CJ, Quill TE, et al.. Association of an educational program in mindful communication with burnout, empathy, and attitudes among primary care physicians. JAMA 2009 Sep 23; 302 (12): 1284-1293.

41. Anderson VL, Levinson EM, Barker W, Kiewra KR. The effects of meditation on teacher perceived occupational stress, state and trait anxiety, and burnout. Sch Psychol Q 1999; 14 (1): 3-25.

42. Talbot LA, Lumden DB. On the association between humor and burnout. Humor: International Journal of Humor Research 2000; 13(4): 419-428.

43. Nargis A, Khan S. Relationship Between Job-Stress And Burnout: Organizational Support And Creativity As Predictor Variables. Pakistan Journal of Psychological Research 2003 Winter; 18 (3/4): 139-149.

44. Arnetz BB, Lucas T.Arnetz J.Organizational Climate, Occupational Stress, and Employee Mental Health: Mediating Effects of Organizational Efficiency. J Occup Environ Med 2011 Jan; 53 (1): 34.

45. Cheung F, Tang C.The influence of emotional dissonance on subjective health and job satisfaction: Testing the stress-strain-outcome model. J Appl Soc Psychol 2010 Dec; 40 (12): 3192-3217.

46. Ybema, Jan F; Smulders, Peter G W; Bongers, Paulien M.Antecedents and consequences of employee absenteeism: A longitudinal perspective on the role of job satisfaction and burnout. European Journal of Work and Organizational Psychology 2010 Feb; 19 (1): 102.

47. Wu YC. Job Stress and Job Performance Among Employees in the Taiwanese Finance Sector: The Role of Emotional Intelligence. Soc Behav Pers 2011; 39 (1): 21-32.

48. Rennesund AB, Saksvik PO. Work performance norms and organizational efficacy as cross-level effects on the relationship between individual perceptions of self-efficacy, overcommitment, and work-related stress. European Journal of Work and Organizational Psychology 2010 Dec; 19 (6): 629-653.

49. Podsakoff NP, LePine JA, LePine MA. Differential Challenge Stressor-Hindrance Stressor Relationships with Job Attitudes, Turnover Intentions, Turnover, and Withdrawal Behavior: A Meta-Analysis. J Appl Psychol 2007; 92(2): 438-454

50. Shirom A, Melamed S. Does burnout affect physical health? A review of the evidence. In: Antoniou ASG Cooper CL. Research companion to organizational health psychology. New horizons in management. (pp.). Northampton, MA, US: Edward Elgar Publishing; 2005. p. 599-622.

51. Okuno K; Yoshimura R, Ueda N, Ikenouchi-Sugita A, Umene-Nakano W, Hori H, ; Hayashi K, Katsuki A, Chen HI, Nakamura J,et al. Relationships between stress, social adaptation, personality traits, brainderived neurotrophic factor and 3-methoxy-4-hydroxyphenylglycol plasma concentrations in employees at a publishing company in Japan. Psychiatry Res 2011 Apr 30; 186 (2-3): 326-332.

52. Larsman $\mathrm{P}$, Lindegård A, Ahlborg G. Longitudinal relations between psychosocial work environment, stress and the development of musculoskeletal pain Stress Med 2011 Aug; 27 (3): 228

53. Tsutsumi A, Kayaba K, Ishikawa S. Impact of occupational stress on stroke across occupational classes and genders. Social Science \& Medicine 2011 May; 72 (10): 1652.

54. Belkic K., Landsbergis PA, Schnall PL, Baker D. Is job strain a major source of cardiovascular disease risk? Scandinavian Journal of Work \& Environmental Health 2004; 30(4): 85-128.

55. Danhof-Pont MB, van Veen T, Zitman FG. Biomarkers in burnout: A systematic review. J Psychosom Res 2011 Jun; 70 (6): 505-524.

56. Sillars A, Parry D. Stress, cognition, and communication in interpersonal conflicts. Communic Res 1982 Apr; 9 (2): 201-226

57. Westman M. Stress and Strain Crossover. Human Relations 2001 Jun; 54 (6): 717-751.

58. Bakker AB, Demerouti E, Schaufeli WB. The crossover of burnout and work engagement among working couples. Human Relations 2005 May; 58 (5): 661-689.

59. Bakker AB, Westman M, Schaufeli WB. Crossover of burnout: An experimental design. European Journal of Work and Organizational Psychology 2007 Jun; 16 (2): 220-239.

60. Rodríguez-Carvajal R. Emotion regulation and social interaction process in resident physician burnout. Ediciones Universidad Autónoma, Madrid; 2007.

61. Quick JC, Quick JD, Nelson DL, Hurrell JJ. In: Quick JC, Quick JD, Nelson DL, Hurrell JJ, editors. Preventive stress management in organizations. American Psychological Association; 1997. p 89-110.

62. Moreno-Jiménez B, Garrosa E, Rodríguez-Carvajal R, Martínez M, Ferrer R. El Burnout del Profesorado Universitario y las Intenciones de Abandono: Un Estudio Multi-Muestra. Revista de Psicología del trabajo y de las organizaciones 2009; 25(2): 149-163.

63. Nahrgang JD, Morgeson FP, Hofmann DA. Safety at work: A meta-analytic investigation of the link between job demands, job resources, burnout, engagement, and safety outcomes. J Appl Psychol 2011 Jan; 96 (1): 71-94. 
64. Schaufeli W, Maslach C, Marek T.Professional Burnout: Recent developments in theory and research. Washington, DC: Taylor \& Francis Publishers; 1993.

65. Harrison RV. Person-environment fit and job stress. In C. L. Cooper and R. Payne (Eds.), Stress at work New York: Wiley; 1978. p. 175-205.

66. Beehr TA, Newman JE. Job stress, employee health, and organizational effectiveness: A facet analysis, model and literature. Personnel Psychology; 1978. 31:665-699.

67. McGrath JE, Beehr TA. Time and the Stress Process: Some Temporal Issues in the Conceptualization and Measurement of Stress. Stress Med 1990; 6: 93-104.

68. Edwards JR. A cybernetic theory of stress, coping, and well-being in organizations. Acad Manage Rev 1992; 17: 238-274.

69. Emmons RA. Personal strivings: An approach to personality and subjective well-being. J Pers Soc Psychol 1986; 51: 1058-1068.

70. Doest LT, Maes S, Gebhardt WA, Koelewijn H. Personal Goal Facilitation through Work: Implications for Employee Satisfaction and Well-Being Applied Psychology 2006 Apr; 55 (2): 192-219.

71. Meurs JA, Perrewé PL. Cognitive activation theory of stress: An integrative theoretical approach to work stress. J Manage 2011 Jul; 37 (4): 1043-1068.

72. Ursin H, Eriksen HR. The cognitive activation theory of stress. Psychoneuroendocrinology 2004 Jun; 29 (5): 567-592.

73. Demerouti E, Bakker AB, Nachreiner F, Schaufeli WB. The job demands-resources model of burnout. J Appl Psychol 2001; 86: 499 -512.

74. Hockey G, Robert J.Cognitive-energetical control mechanisms in the management of work demands and psychological health. In: Baddeley AD, Weiskrantz L. Attention: Selection, awareness, and control. New York, NY, US: Clarendon Press/Oxford University Press, 1993 p.328-345.

75. Xanthopoulou D, Bakker A, Demerouti E, Schaufeli W. The role of personal resources in the Job Demands-Resources Model. Int J Stress Manag 2007; 14 (2): 121-141.

76. Hobfoll SE. Conservation of resources: a new attempt at conceptualizing stress. Am Psychol 1989; 44(3): 513-524.

77. Hobfoll SE, Shirom A. Conservation of resources theory: application to stress and managemente in the workplace. In: Golembiewski RT. Handbook of organizational behavior (2nd. ed, rev. ed and, exp.ed.). New York, NY, US: Marcel Dekker; 2001.

78. Siegrist J. Adverse health effects of high effort-low reward conditions at work. J Occup Health Psycholm 1996; 1: 27-43.

79. Moreno-Jiménez B, Alonso M, Álvarez E. Sentido de coherencia, personalidad resistente, autoestima y salud. Revista de Psicología de la Salud, 1997;9:115-138.

80. Lazarus RS. Emotion and Adaptation. New York: Oxford University Press; 1991.

81. Edelwich J, Brodsky A. Burnout States of Disillusionment in the Helping Profession, New York, NY: Human Services Press; 1980

82. Ridge M, Wells J, Denny M, Cunningham J, Chalder T. Developing a web-based stress management intervention for occupational support workers. J Ment Health, 2011;20: 185.

83. Leung SS, Chiang VCL, Chui Y, Lee, ACK, Mak, Y. Feasibility and potentials of online support for stress management among secondary school teachers. Stress Health, 2011;27: 282

84. Bond FW, Flaxman PE, van Veldhoven MJPM, Biron M. .The impact of Psychological Flexibility and Acceptance and Commitment Therapy (ACT) on Health and Productivity at Work. In Houdmont J, Leka S, editors.Contemporary Occupational Health Psychology. Global perspectives on research and practice Volume 1. Chichester,West Sussex, UK: Wiley-Blackwell; 2010. p 296-313

85. Hayes SC, Strosahl KD, Wilson KG. Acceptance and commitment therapy: An experiential approach to behavior change. New York, NY, US: Guilford Press; 1999.

86. Marianetti $\mathrm{O}$, Passmore J. Mindfulness at work: Paying attention to enhance well-being and performance. In Linley PA, Harrington S, Garcea N, editors.Oxford handbook of positive psychology and work. New York, NY, US: Oxford University Press 2010. p. 189-200.

87. Vilardaga R, Luoma JB, Hayes SC, Pistorello J, Levin ME, Hildebrandt MJ, Kohlenberg B, Roget NA, Bond $\mathrm{F}$, et al. Burnout among the addiction counseling workforce: The differential roles of mindfulness and values-based processes and work-site factors. J Subst Abuse Treat 2011 Jun; 40 (4): 323-335.

88. Flaxman PE, Bond FW. Acceptance and commitment training: Promoting psychological flexibility in the workplace. In Baer RA, editor. Assessing mindfulness and acceptance processes in clients: Illuminating the theory and practice of change. Oakland, CA, US:Context Press/New Harbinger Publications; 2010. P 282-306. 
89. Berceli D, Napoli M.A proposal for a mindfulness-based trauma prevention program for social work professionals. Complement Health Pract Rev 2006; 11(3): 153-165.

90. Ruiz CO, Rios FL, Martin SG. Psychological intervention for profesional burnout in the Palliative Care Unit at Gregorio Marañon University Hospital. Medicina Preventiva 2008; 15 (2): 93-97.

91. Brinkborg H, Michanek J, Hesser H, Berglund G. Acceptance and commitment therapy for the treatment of stress among social workers: A randomized controlled trial. Behav Res Ther 2011 Jun; 49 (6-7): 389-398.

92. Flaxman PE, Bond FW. Worksite stress management training: Moderated effects and clinical significance. J Occup Health Psychol 2010 Aug; 15 (4) : 347-358.

93. Flaxman PE, Bond FW. A randomised worksite comparison of acceptance and commitment therapy and stress inoculation training. Behav Res Ther 2010 Aug; 48 (8): 816-820.

94. Atkinson W. Turning Stress into Strength. HRMagazine 2011 Jan; 56 (1): $49-52$.

95. Hahn VC, Binnewies C, Sonnentag S, Mojza EJ. Learning how to recover from job stress: Effects of a recovery training program on recovery, recovery-related self-efficacy, and well-being. J Occup Health Psychol 2011 Apr; 16 (2): 202-216.

|||||||||||||||||||||||||||||||||||||||||||||||||||||||||||||||||||||||||||||||||||||||||||||||||||||||||||||||||||||||||||||||||||||||||||||||||||||||||||||||||||||||||||||||||||||||||||||| 